\title{
Application of English Assisted Instruction based on Mobile Learning Qinyuan Hui
}

\author{
Zhengzhou university of industrial technology.Henan.China
}

Keywords: Mobile learning;Cloud server;English computing

\begin{abstract}
The limitation of terminal equipment and the scarcity of mobile learning resource are key constrains of mobile learning development. However, English computing supplies a new way of solving the problem. For English computing, the calculation and the storage of task are conducted in the cloud server. As a result, computing and storage work of mobile phone are separated from the machine. So user can customize through English computing services according to personal need. Thus a lot of works that are realized only in high-performance computers before, but now can be finished through the mobile phones with poor configuration. This paper through theoretical study and based on the theory of mobile learning and English computing, it points out the disadvantages of mobile learning and puts forward the solution of English computing technology.
\end{abstract}

\section{Introduction}

With the rapid development of computer technology and network technology, the learning mode in the environment of information technology changes constantly. The occurrence of new technology in the education field brings new learning methods and learning experience. So are the Computer Assisted Instruction, the Distance Education and the Computer Network Education[1]. Owning to the development of wireless communication network and mobile terminal equipment, the application scope of the Computer Network Education are greatly expanded and learning activity are also developed from PC to intelligent terminal. Those result in the appearance of a new study mode-Mobile Learning. Without the limitations of time and space, Mobile Learning enables learners to study at any time and in any place[2]. Mobile learning has more advantages than traditional learning mode in autonomy, convenience, flexibility, interactivity and continuity[3]

At present,mobile learning with the support of English technology is still in the initial exploration stage, but its unique advantages indicate that English has a good potential application in the field of mobile learning. Mobile learning can combine the new technology of multimedia with mobile devices, plus the haptic interaction, smart environment, wireless communication and so on, which can combine them together into the field of education and training. Therefore, mobile learning has showed different digital learning characteristic, which is quite different from the general characteristics of the traditional fixed wired PC pattern based on the computer network[4][5].

\section{The Overview of English Assisted Instruction}

\subsection{English}

English is a virtual resource pool based on needs, it can distribute the computing tasks in the resource pool, which can make all application systems obtain a variety of software services, computing power and storage space according to different needs[6]. Then users can achieve the agreed service according to the level of agreement, while the provider of the services can provide service with pay according to different agreements. The pattern of English computing model is different from the traditional calculation pattern which takes PC machine as the center, moreover, its data and programs are no longer stored in the personal computer[7]. However, they are stored in the Internet data center, which is so called "cloud" data. Then users do not need the traditional desktop software and disk space, as well as its processing power. As long as users can have access 
to the Internet, they can visit "cloud" services at any time and any sites, which can be shown in Fig.1.

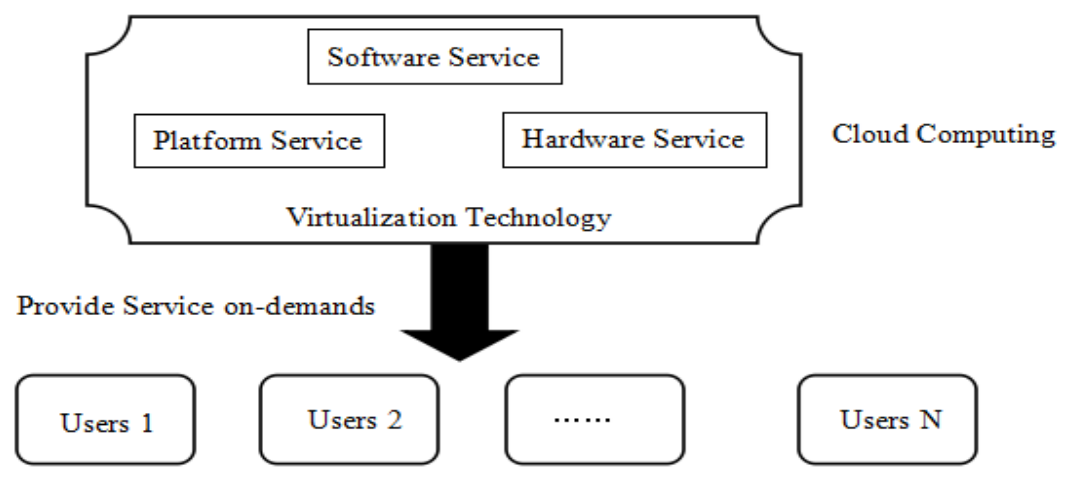

\subsection{English computing Assisted Instruction}

Fig.1: Schematic Diagram of English Model

English computing Assisted Instruction (CCAI) refers that the schools and the teachers make use of the "English" to provide education with "clouds service", so as to build a personalized and information-based teaching environment, which can support teachers and students with the effective teaching and learning, promote the learner's wisdom development as well as the development of the advanced thinking ability, finally, in order to improve the quality of educating and teaching[8].

\section{Critical learning Calculations}

In a cloud environment, the critical learning modeling that oriented the data mining can learn from the critical learning method in project management. According to specific data mining workflow, combined with the control flows between tasks and the input-output relationship, the workflow was abstracted into DMTOE (Data Mining Task on Edge) network, after obtained the critical learning of the data mining workflow, based on multiple examples pricing model of cloud resource, it carries out the multi examples portfolio purchase.

In DMTOE network, the vertices represent events, directed edges represent tasks, the weights on the edge represent the duration of the task. The node without predecessor node was called the entrance node, and the node without the successor node was called the exit node. There are multiple entrance (exit) nodes in some task graph, and they can be connected to an entrance (exit) node by the edge with the weights of 0 . The directed edges described the sequence between the tasks. The node cannot be executed before receiving the message from the successor nodes and a return message from the predecessor nodes.

We assume that the data mining workflow has a total of 15 tasks, 10 data sets, 5 data centers, the dependence relationship between tasks and the call relations between data and tasks, as shown in figure 2 .

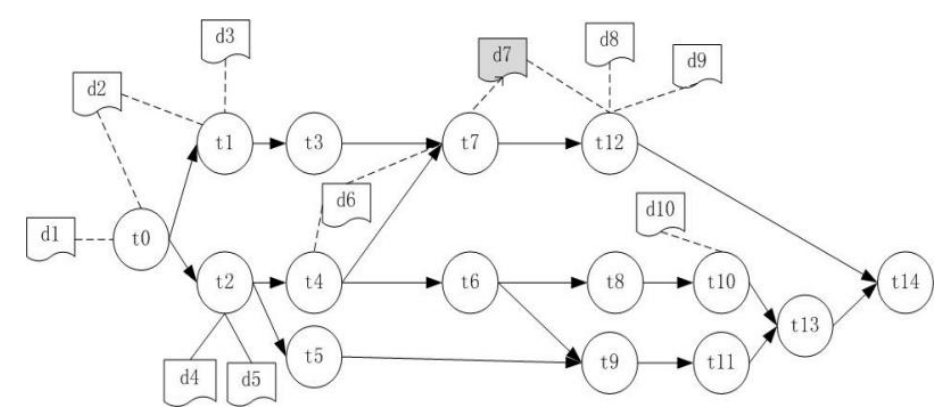

Figure2. Data Mining Workflow

First, put the data set aside, only analyze the input-output the relationship of the data mining tasks, as shown in figure 3. 


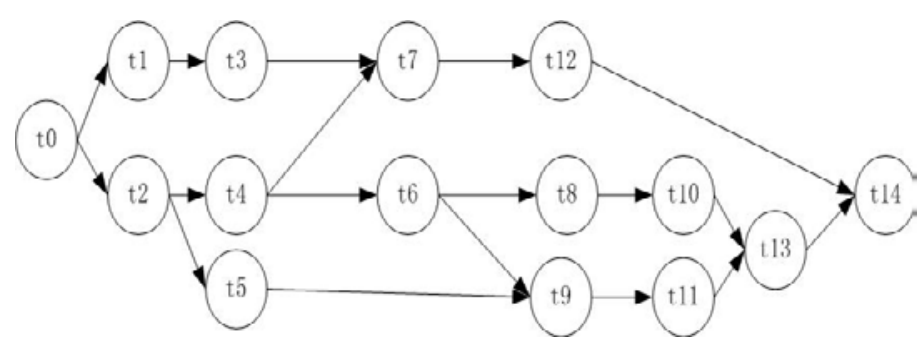

Figure3. Task Dependency Graph

\section{Theory of Mobile Learning}

\subsection{Interpretation of Mobile Learning (M-learning)}

Mobile learning is to use the mobile devices to learn, which is occurred in the context of learning. It is a kind of mixed learning pattern with other ways of learning modes[9]. However, mobile learning is not simply push content to the small screen through mobile devices, but is focused on the execution driven by the efficient learning process. Mobile learning is the product of mobile communication technology combined with the development of computer network. The intelligent and portable mobile terminal equipment is regarded as an extension of the technology of digital learning.

\subsection{Resources Design of Mobile Learning}

Mobile learning resources are designed according to the learning objectives, which can show specific learning contents, reactions with certain teaching strategies. As shown in fig 4. The resources for each course are composed of a variety of media materials, including video, audio course, e-book, images, animation, etc. Through the support of a variety of media materials, it can make the clients and users gain multiple intellectual stimulation by using mobile learning terminals, in order to achieve the learning effect. Among them, the content of video course takes 5 or 10 minutes as a module, the content of course takes skills learning as segmentation, there is loose connection among courses; Besides, the content of text takes each knowledge point as various points of different courses, which is closely linked to the content and its form is relatively independent; pictures can support formats such as jpeg, bmp, gif and other common used formats, while audio files can support MP3, WMA and some other forms.

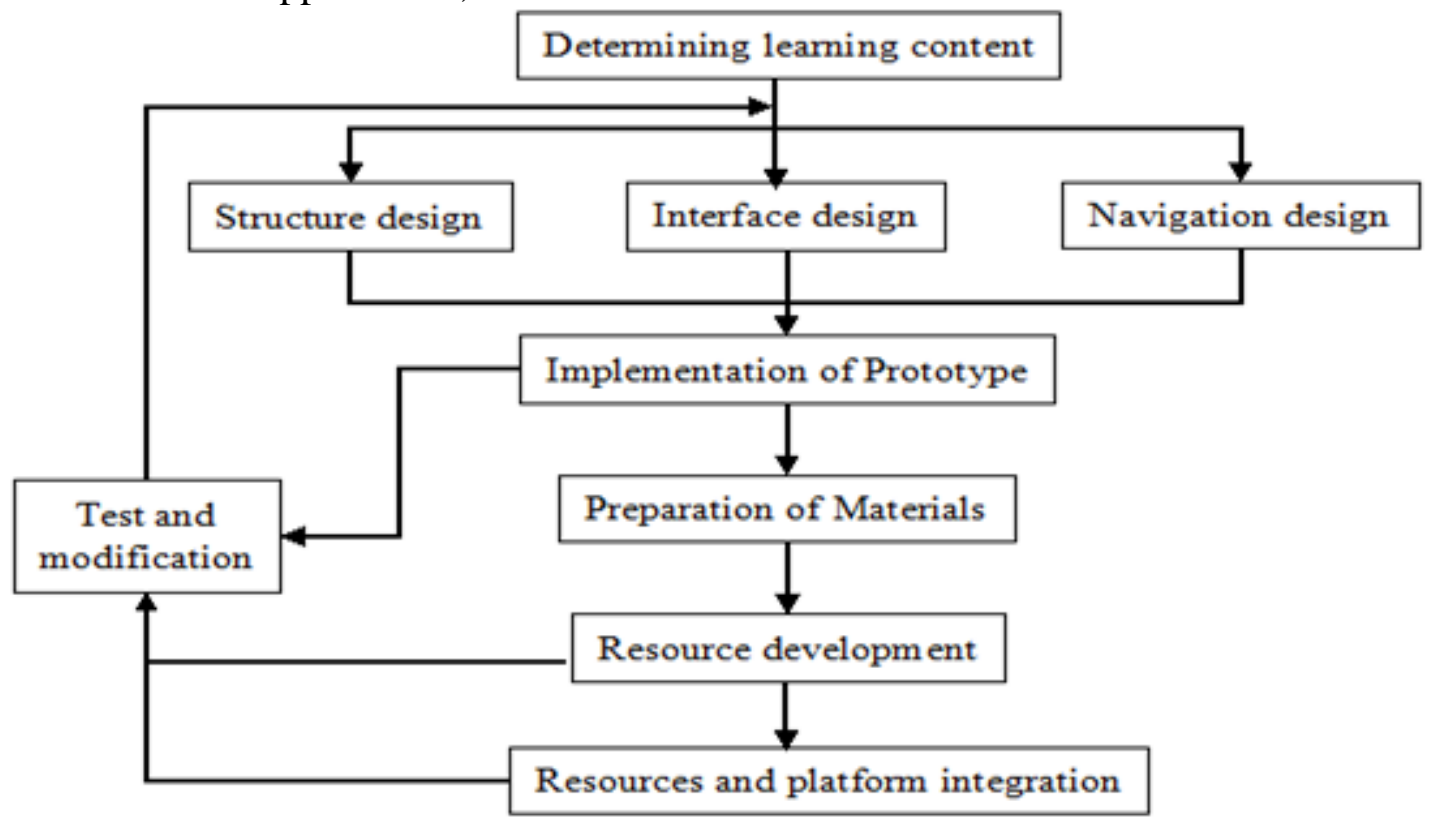

Fig.4 Design and Development of Mobile Learning Resources based on English

\section{Conclusion}

This paper explored the design of mobile learning system based on English, in an effort to realize the combination of new technology and advanced learning concept, hoping to meet the 
requirements of people who can get information freely at anyplace and anytime, with personalized and diversified mobile learning contents, so as to improve the quality of education and realize the balanced development of education, cultivating more high-quality talents, so as to provide new strategies for the education and training industry. Although the construction of mobile learning system based on English existed many problems, with the English technology and application becoming more and more mature, the mobile learning system with the support of English technology will show strong vitality in the near future, which will have a profound effect on the field of education.

\section{REFERENCES}

[1] HEW ITT C. 2008. ORGs for scalable, robust privacy-friendly client English computing. IEEE Internet Computing, vol.125, pp 96-99.

[2] Vaquero L, Rodero-Marino L, Caceres J. 2009. A break in the clouds: towards a cloud definition. SIGCOMM Computer Communication Review, vol.139, pp50-55.

[3] Chin-Chung Tsaia, Sunny S.J.Lina, Shyan-Ming Yuan. 2002. Developing science activities through a networked peer assessment system. Computers\&Education, vol.123, pp243-244.

[4] Barnum, C, Parman, W. 2002. Bringing introduction to the teacher: A blended learning Model. T.H.E Journal. vol.130, pp56-64.

[5] Bersin J. 2004. The Blended Learning Book:Bestpraetiees, proven Methodlog And Lessons Learned. SanFraneiseo: Peiffer Publishing. pp50- 51.

[6] Y. Geng, J. He, K. Pahlavan, Modeling the Effect of Human Body on TOA Based Indoor Human Tracking, International Journal of Wireless Information Networks 20(4), 306-317 